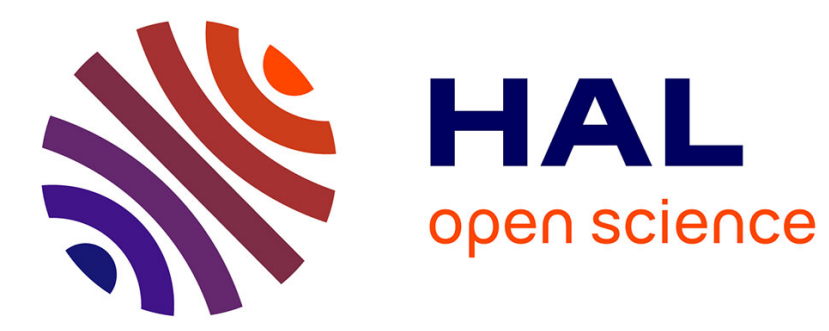

\title{
Etude du mode de répartition du courant dans un contact électrique glissant multifilamentaire
}

L. Boyer

\section{To cite this version:}

L. Boyer. Etude du mode de répartition du courant dans un contact électrique glissant multifilamentaire. Revue de Physique Appliquée, 1985, 20 (3), pp.183-190. 10.1051/rphysap:01985002003018300 . jpa-00245321

\section{HAL Id: jpa-00245321 https://hal.science/jpa-00245321}

Submitted on 1 Jan 1985

HAL is a multi-disciplinary open access archive for the deposit and dissemination of scientific research documents, whether they are published or not. The documents may come from teaching and research institutions in France or abroad, or from public or private research centers.
L'archive ouverte pluridisciplinaire HAL, est destinée au dépôt et à la diffusion de documents scientifiques de niveau recherche, publiés ou non, émanant des établissements d'enseignement et de recherche français ou étrangers, des laboratoires publics ou privés. 
Classification

Physics Abstracts

84.70

\title{
Etude du mode de répartition du courant dans un contact électrique glissant multifilamentaire
}

\author{
L. Boyer \\ Laboratoire de Génie Electrique de Paris (L.G.E.P.) (*), Universités de Paris VI et Paris XI, GRECO 40 « Contacts \\ Electriques », Ecole Supérieure d'Electricité, Plateau du Moulon, 91190 Gif sur Yvette, France
}

(Reçu le 30 janvier 1984, révisé les 8 juin et 8 novembre, accepté le 30 novembre 1984)

\begin{abstract}
Résumé. - Après avoir décrit une version moderne de contact électrique glissant multifilamentaire, on montre pour quelle raison le courant peut se répartir inégalement entre les différents filaments constituant un balai. On présente ensuite une modélisation dans laquelle la région de partage du courant est assimilée à un assemblage de couches co-axiales de conductivités différentes. On montre, grâce à ce modèle, que la répartition du courant est analogue à celle due à l'effet de peau, dans un matériau massif parcouru par du courant alternatif. On décrit enfin une expérience dont les résultats sont en bon accord avec les résultats tirés de la modélisation.
\end{abstract}

\begin{abstract}
After having described a multifilamentary electric sliding contact of a new kind, we give the reason for which the current may be unequally shared among the filaments composing a brush. Then, we present a model in which we assume that the current sharing domain is constituted by an assembly of shells of different conductivities. By using this model, we show that the current sharing in a brush is similar to that due to the skin effect in a bulk material transporting an alternating current. We finally describe an experiment whose results are in good agreement with the theoretical predictions.
\end{abstract}

\section{Introduction.}

Depuis quelques années, plusieurs laboratoires s'intéressent, de nouveau, aux contacts électriques glissants multifilamentaires $[1,9]$. La première description de ce type de contact, qui remonte à un siècle, est due à Thomas Edison [10]. C'est d'ailleurs depuis cette époque que l'on utilise, par abus de langage, le terme de balai pour désigner les contacts électriques glissants monolithiques en carbone. En ce qui concerne leur forme actuelle [11], ces balais sont constitués par des filaments métalliques dont le diamètre est compris entre $20 \mu \mathrm{m}$ et $150 \mu \mathrm{m}$ et dont la longueur varie entre quelques $\mathrm{mm}$ et quelques dizaines de $\mathrm{mm}$. Différents métaux, comme le cuivre, les alliages de cuivre ou l'or, sont utilisés pour réaliser les filaments. Les pistes sur lesquelles frottent ces balais sont en cuivre et peuvent avoir subi un traitement de surface dont le but est d'améliorer les comportements tribologique et électrique de l'ensemble. Enfin, l'atmosphère dans laquelle fonctionnent ces contacts doit être telle qu'il ne puisse pas se former, à l'interface filament/piste, une barrière

(*) Laboratoire associé au C.N.R.S., No 127. semi-conductrice ou isolante, difficilement franchissable (par effet tunnel) par les électrons. Ainsi, si on se restreint, pour des raisons pratiques, au cas de filaments en alliage de cuivre $(\mathrm{Cu}-1,2 \% \mathrm{Cd}$ par exemple), frottant sur une piste dorée, le fonctionnement de ces balais dans l'atmosphère ambiante, est rendu impossible par la formation d'une couche semi-conductrice d'oxyde de cuivre à l'extrémité des filaments. On doit donc utiliser un gaz non réactif comme l'azote $[8,9]$ ou le dioxyde de carbone $[2,7]$ que l'on peut, éventuellement, humidifier pour « lubrifier" le contact. Dans ce cas, ces balais peuvent transporter des densités de courant très importantes (supérieure à $100 \mathrm{~A} \mathrm{~cm}^{-2}$ ) sous des d.d.p. de contact relativement faibles $(50$ à $200 \mathrm{mV})$, avec des vitesses de glissement atteignant $100 \mathrm{~ms}^{-1}$ et des taux d'usure (longueur usée/distance parcourue) de l'ordre de $10^{-11}$.

La réalisation des balais comporte deux étapes techniques délicates. D'abord, il faut pouvoir disposer de fil de faible diamètre $(50 \mu \mathrm{m}$ au L.G.E.P.) rectiligne. Comme il est impossible de parvenir à ce but par les techniques de tréfilage classiques, nous avons réalisé, au L.G.E.P., une machine [12] permettant de dresser le fil. Ensuite, il s'agit d'assembler conve- 
nablement les filaments entre eux pour réaliser un balai en comportant plusieurs milliers (3000 actuellement au L.G.E.P.). L'assemblage des filaments doit être de bonne qualité, tant sur le plan mécanique que sur le plan électrique. Pour réaliser cette opération, nous avons d'abord utilisé une technique de soudage à basse température $\left(T \simeq 220^{\circ} \mathrm{C}\right)$ que nous avons dû abandonner car elle altérait certaines caractéristiques des filaments. Nous nous sommes alors orientés vers une technique de sertissage à froid qui, bien que donnant des résultats satisfaisants, présente un inconvénient. Cet inconvénient est schématisé sur la figure 1 qui représente de manière idéalisée une coupe partielle de l'assemblage des filaments au niveau de la partie sertie. Sur cette figure, on a représenté, de manière approchée, un cheminement possible pour les lignes de courant. On constate que ce cheminement s'effectue de filament à filament par l'intermédiaire de constrictions. Ces constrictions opposent au passage du courant une résistance d'autant plus grande que leur surface est faible. Par ailleurs, elles peuvent avoir la structure MétalIsolant-Métal ou Métal-Semi-Conducteur-Métal, ce qui introduit une résistance supplémentaire.

Le but de cette étude est de montrer, théoriquement et expérimentalement, que le mode d'assemblage des filaments conduit à une répartition inégale du courant (continu) semblable à ce que l'on observe en régime alternatif avec l'effet de peau classique dans les matériaux massifs.

\section{Modélisation d'un balai.}

Les différentes parties d'un balai sont représentées à l'échelle sur la figure $2 \mathrm{a}$. La modélisation a pour objectif de définir la résistance $R_{\mathrm{b}}$ du balai

$$
R_{\mathrm{b}}=\frac{V}{I}
$$

$V$ étant la tension appliquée et $I$ le courant injecté (Fig. 2a), et de faire apparaître les paramètres dont dépend $R_{\mathrm{b}}$. Il est clair que pour modéliser de façon précise la partie $A$ du balai, définie sur la figure $2 a$, il faut considérer pour cette région qui est représentée en coupe sur la figure 1, un matériau à conductivité électrique anisotrope $\left(\sigma_{\mathrm{z}} \gg \sigma_{\mathrm{r}}\right)$. Sans négliger cette voie (au deuxième ordre) sur laquelle nous travaillons, nous présentons ici un modèle (au premier ordre) dans lequel la partie A du balai est représenté (Fig. 2b) par un assemblage de couches équipotentielles infiniment conductrices, en contact, par l'intermédiaire de couches infiniment fines, caractérisées par une résistance surfacique $R_{\mathrm{s}}$ constante (indépendante du $\mathrm{n}^{\circ}$ de la couche) relative à des lignes de courant radiales.

L'expression de la surface $S_{\mathrm{A}}(r)$ entre deux couches infiniment conductrices de rayons voisins de $r$ s'écrit :

$$
S_{\mathrm{A}}(r)=2 \pi r L_{\mathrm{A}}
$$

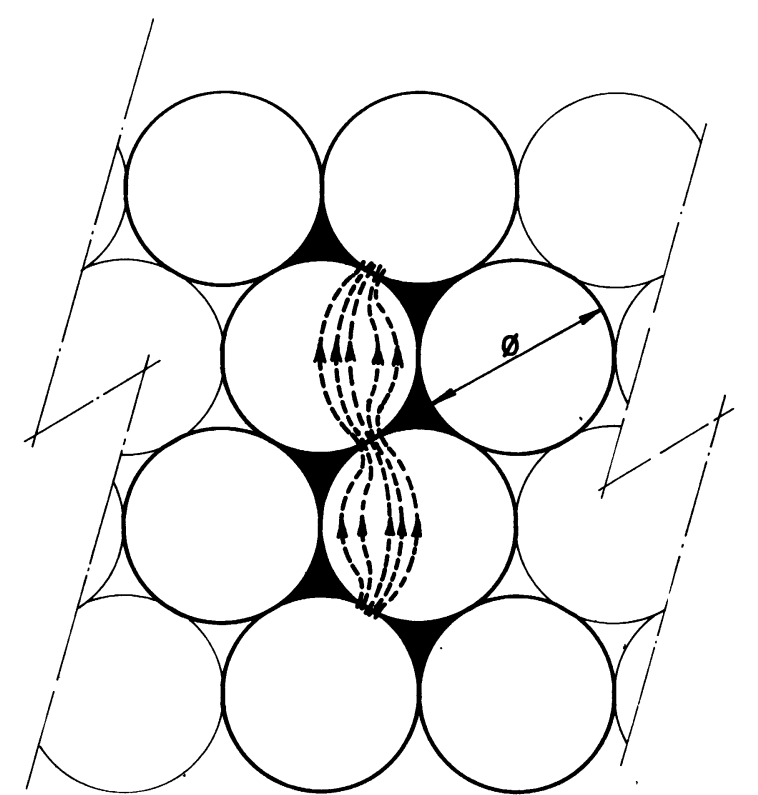

Fig. 1. - Allure des lignes de courant radiales dans la partie sertie du balai.

[Sketch of the radial current lines in the crimped part of the brush.]

avec $L_{\mathrm{A}}$ : longueur de la partie A du balai. Dans ces conditions, la résistance $R_{\mathrm{p}}(r)$ entre ces deux couches vaut :

$$
R_{\mathrm{p}}(r)=\frac{R_{\mathrm{s}}}{S_{\mathrm{A}}(r)}=\frac{R_{\mathrm{s}}}{2 \pi r L_{\mathrm{A}}}
$$

Si l'on suppose que toutes les couches conductrices sont caractérisées par la même épaisseur $\phi$, égale au diamètre d'un filament, et le même coefficient de foisonnement $f$, le nombre $n(r)$ de filaments de la couche de rayon moyen $r$ est donné par :

$$
n(r)=f \frac{2 \pi r \phi}{\frac{\pi \phi^{2}}{4}}=\frac{8 f r}{\phi} .
$$

Si la résistance d'un filament, relative à la partie $B$ définie sur la figure 2a, est supposée égale à $R$, la résistance $R_{\mathrm{c}}(r)$ entre la couche infiniment conductrice de rayon $r$ et la piste sera donnée par :

$$
R_{\mathrm{c}}(r)=\frac{R}{n(r)}=\frac{R \phi}{8 f r} .
$$

On remarque alors que $R_{\mathrm{p}}(r)$ et $R_{\mathrm{c}}(r)$ varient comme $1 / r$, ce qui permet, en faisant le rapport de ces deux grandeurs, de définir un nombre sans dimension $\gamma$ :

$$
\gamma=\frac{R_{\mathrm{p}}(r)}{R_{\mathrm{c}}(r)}=\frac{4 f R_{\mathrm{s}}}{\pi L_{\mathrm{A}} R \phi}
$$

qui est d'autant plus petit que le contact entre couches est de bonne qualité. 

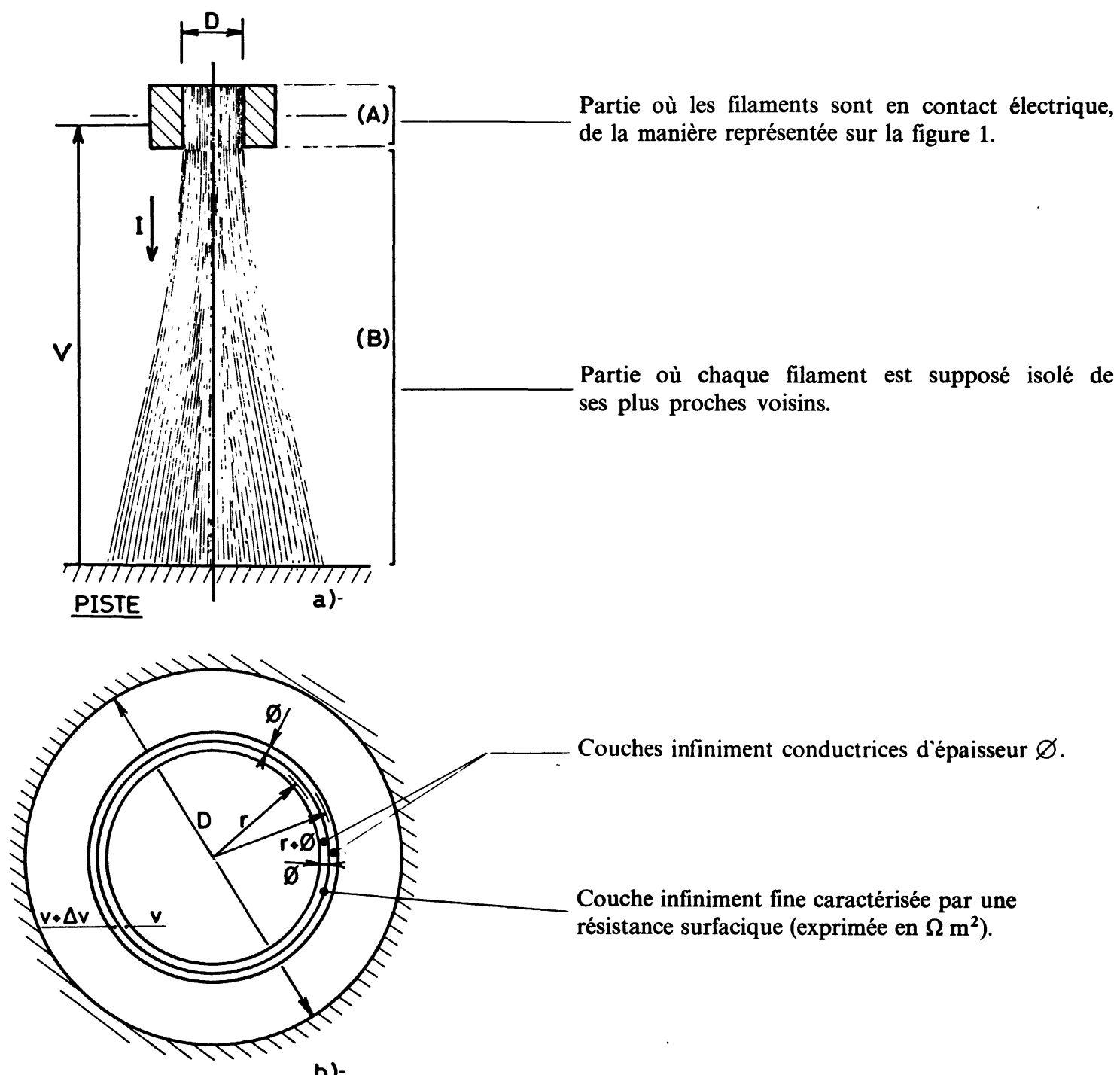

b)-

Fig. 2. - a) Coupe longitudinale d'un balai. b) Modélisation de la partie A d'un balai.

[a) Longitudinal section of a brush. b) Modelling of the portion A of a brush.]

Dans le cadre de notre étude, on peut, en tenant compte des considérations précédentes, modéliser un balai par un circuit en échelle semblable à celui qui est représenté sur la figure 3 . Les équations électriques relatives à l'élément de ce circuit compris entre $r$ et $r+\phi$ s'écrivent :

$$
\begin{gathered}
\Delta v(r)=R_{\mathrm{p}}(r) i(r)=\frac{\gamma \phi R}{8 r f} i(r) \\
\Delta i(r)=\frac{v(r)}{R_{\mathrm{c}}(r)}=\frac{8 r f}{\phi R} v(r) .
\end{gathered}
$$

De façon à obtenir des équations différentielles, plus maniables que (7) et (8), nous divisons les deux membres de ces équations par $\phi$ et nous effectuons les «passages à la limite » suivants :

$$
\begin{aligned}
\frac{\Delta v}{\phi} & \rightarrow \frac{\mathrm{d} v}{\mathrm{~d} r} \\
\frac{\Delta i}{\phi} & \rightarrow \frac{\mathrm{d} i}{\mathrm{~d} r}
\end{aligned}
$$

que l'on peut justifier en remarquant que le diamètre d'un filament est toujours très petit devant celui du balai. Dans ces conditions, on obtient le système d'équations couplées suivant :

$$
\begin{aligned}
& \frac{\mathrm{d} v}{\mathrm{~d} r}=\frac{\gamma R}{8 r f} i \\
& \frac{\mathrm{d} i}{\mathrm{~d} r}=\frac{8 r f}{\phi^{2} R} v .
\end{aligned}
$$




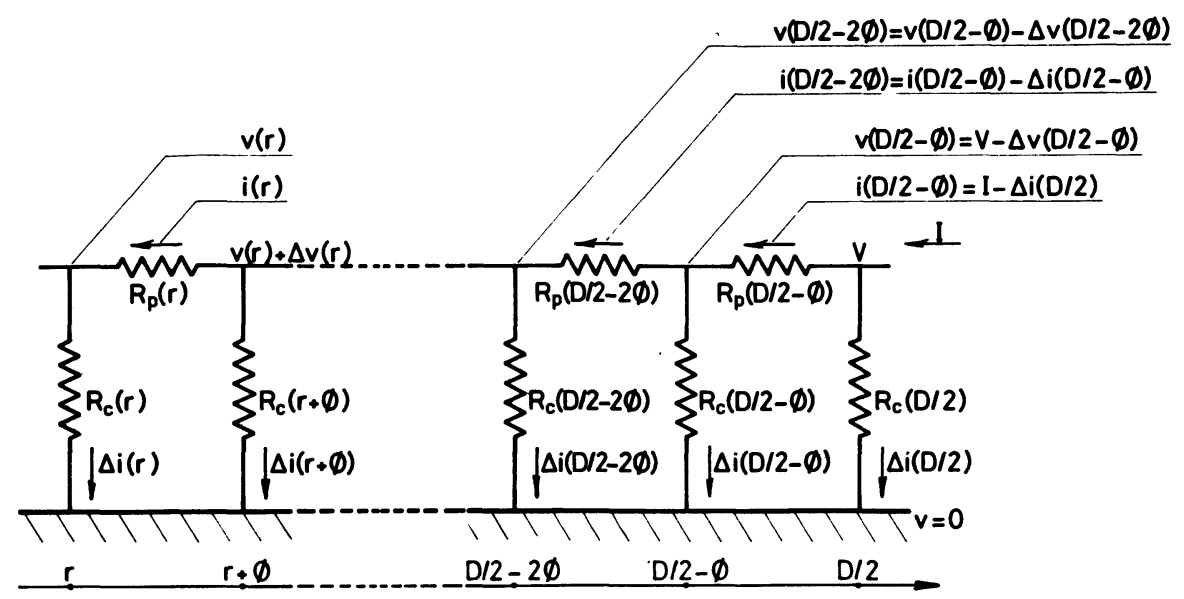

Fig. 3. - Réseau en échelle utilisé pour modéliser un balai.

[Ladder network used to model a brush.]

Ensuite, on reporte (11) dans (12) et on obtient l'équation différentielle du second ordre qui régit les variations de $v$ :

$$
\frac{\mathrm{d}^{2} v}{\mathrm{~d} r^{2}}+\frac{1}{r} \frac{\mathrm{d} v}{\mathrm{~d} r}-\frac{\gamma v}{\phi^{2}}=0 .
$$

En posant :

$$
\lambda=\frac{\phi}{\sqrt{\gamma}}
$$

et

$$
u=\frac{r}{\lambda}
$$

l'équation s'écrit sous la forme suivante :

$$
\frac{\mathrm{d}^{2} v}{\mathrm{~d} u^{2}}+\frac{1}{u} \frac{\mathrm{d} v}{\mathrm{~d} u}-v=0
$$

qui admet comme solution générale :

$$
v=B I_{0}(u)+C K_{0}(u)
$$

où $I_{0}$ et $K_{0}$ sont des fonctions de Bessel modifiées de première et de seconde espèce d'ordre zéro.

Si on note $D$, le diamètre de la région multifilamentaire du balai au niveau de la partie A et si on impose comme conditions aux limites :

$$
\begin{aligned}
& v(r=D / 2)=V \\
& v(r=0) \text { fini }
\end{aligned}
$$

la solution de (13) se réduit à :

$$
v(r)=V \frac{I_{0}(r / \lambda)}{I_{0}(D / 2 \lambda)}
$$

Ensuite (11) permet d'obtenir l'équation donnant le courant :

$$
i=\frac{V 8 r f}{R \phi \sqrt{\gamma}} \frac{I_{1}(r / \lambda)}{I_{0}(D / 2 \lambda)} .
$$

La relation (21) permet enfin d'obtenir la résistance $R_{\mathrm{b}}(\gamma)$ du balai :

$$
R_{\mathrm{b}}(\gamma)=\frac{V}{I}=R \frac{\phi \sqrt{\gamma} I_{0}(D \sqrt{\gamma} / 2 \phi)}{4 D f I_{1}(D \sqrt{\gamma / 2} \phi)} .
$$

Le cas du «balai idéal " dans lequel le courant se répartit uniformément entre tous les filaments correspond à $\gamma=0$. En effectuant les développements limités de $I_{0}$ et $I_{1}$, on vérifie bien que (22) donne la bonne expression de $R_{\mathrm{b}}(0)$ :

$$
R_{\mathrm{b}}(0)=\frac{R \phi^{2}}{f D^{2}} .
$$

En définissant le nombre $\beta$, sans dimension, de la manière suivante :

$$
\beta=\frac{D \sqrt{\gamma}}{2 \phi}
$$

le rapport $R_{\mathrm{b}}(\gamma) / R_{\mathrm{b}}(0)$ prend la forme simple :

$$
\frac{R_{\mathrm{b}}(\gamma)}{R_{\mathrm{b}}(0)}=\frac{\beta I_{0}(\beta)}{2 I_{1}(\beta)}=S(\beta)
$$

qui exprime la qualité du sertissage. En pratique, il faut que $S(\beta)$ (défini par (25)), ait une valeur aussi proche que possible de l'unité. La figure 4 représente la fonction $S(\beta)$.

\section{Confrontation du modèle avec une expérience.}

3.1 Conditions eXPÉrimentales. - Nous ne nous sommes pas mis dans la situation réelle d'un balai frottant sur une piste (voir Fig. 2a) pour évaluer la validité de notre modèle. En effet, dans ce cas, la 


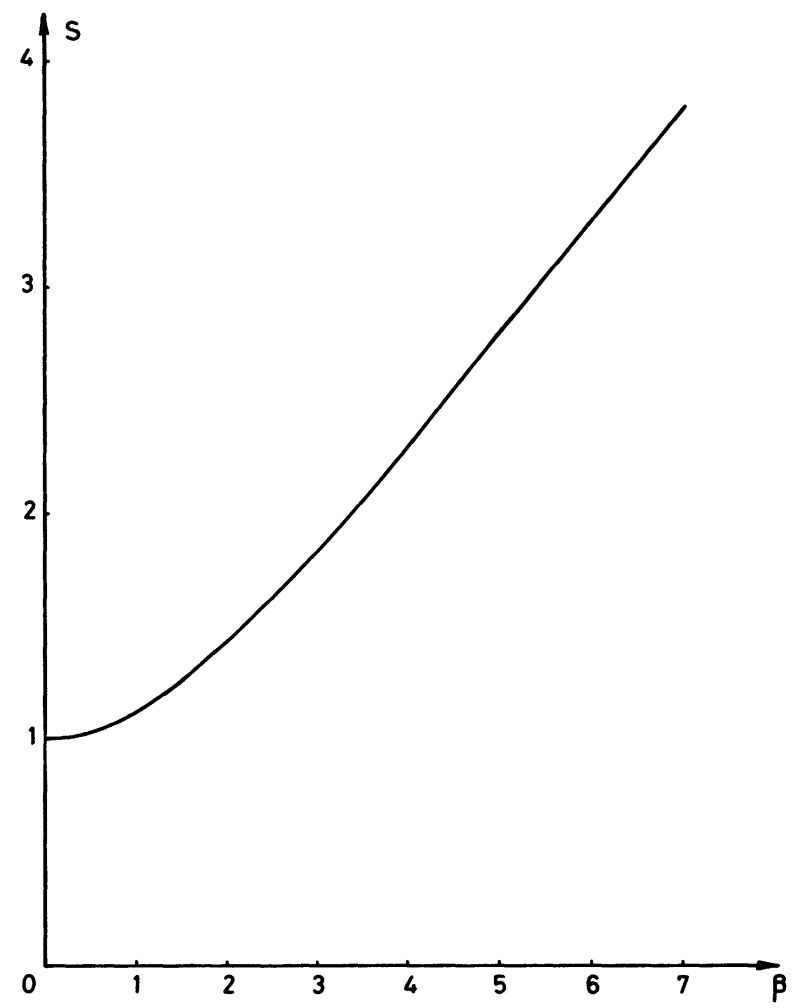

Fig. 4. - Graphe de la fonction $S(\beta)=\frac{\beta}{2} \frac{I_{0}(\beta)}{I_{1}(\beta)}$.

[Plot of the function $S(\beta)$.]

résistance $R$, précédemment définie et intervenant dans (6), doit tenir compte, [13], de l'existence d'une constriction et de celle d'un film à l'interface filament/piste. Comme les contributions de ces deux éléments sont très difficiles à déterminer, nous avons préféré simuler un contact filament/piste parfait, en nous mettant dans la situation décrite sur la figure 5 . Nous avons ainsi réalisé des éprouvettes de longueurs $L$ différentes, serties aux deux extrémités et comportant, chacune, 3000 filaments de $50 \mu \mathrm{m}$ de diamètre, en cuivre au cadmium. Dans ce cas, la résistance $R$ est proportionnelle à $L$. Si on admet, par ailleurs, que la résistance surfacique inter-couches $R_{\mathrm{s}}$ est la même pour tous les sertissages réalisés, on trouve que $\gamma$ et $\beta$ doivent varier respectivement comme $L^{-1}$ et $L^{-1 / 2}$ [voir (6) et (24)]. Dans ces conditions, si notre modèle est représentatif à la réalité, la résistance :

$$
R_{\text {exp. }}(L)=\frac{V(L)}{I}
$$

d'une éprouvette de longueur $L$, ne doit pas être proportionnelle à $L$ mais varier suivant une loi non linéaire. Une bonne manière de mettre en évidence cette non-linéarité, consiste à mesurer la résistance d'un filament puis à rapporter $R_{\text {exp. }}(L)$ à la valeur $R_{\text {idéale }}(L)$ calculée en supposant que le courant se

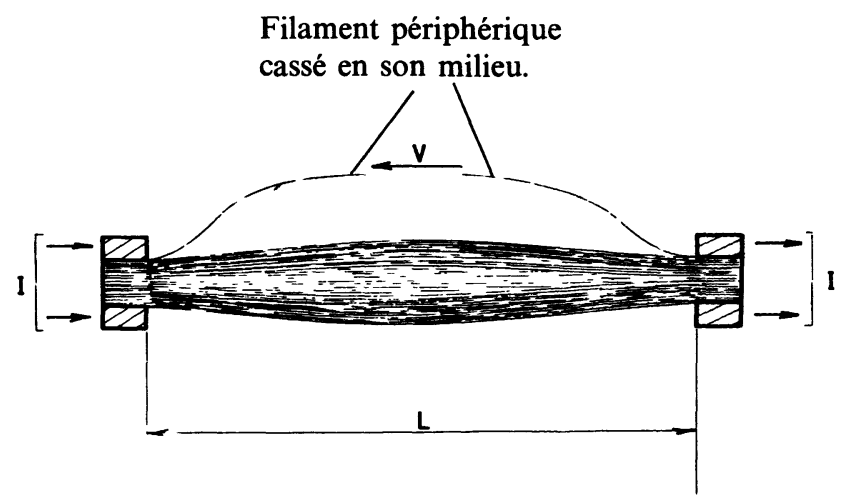

Fig. 5. - Méthode utilisée pour mesurer la résistance d'une éprouvette multifilamentaire.

[Method used for the measurement of the resistance of a multifilamentary test sample.]

répartit uniformément entre tous les filaments. La technique de mesure que nous avons retenue est globale, en ce sens qu'elle fournit la valeur de la résistance d'un ensemble de 3000 filaments et non pas la valeur du courant passant dans chaque filament $\left({ }^{1}\right)$.

Dans nos expériences, le courant est amené à chaque éprouvette par l'intermédiaire de colliers serrant chacune des deux douilles d'extrémités. La différence de potentiel entre les surfaces internes de ces deux douilles est mesurée par l'intermédiaire d'un filament périphérique cassé en son milieu (voir la Fig. 5). La production de fil rectiligne de $50 \mu \mathrm{m}$ de diamètre étant une opération délicate (non exempte de casses), qui doit servir également à la fabrication de balais, nous n'avons réalisé qu'un nombre restreint d'éprouvettes : trois de longueurs $L$ égales à $16 \mathrm{~mm}$. $32 \mathrm{~mm}$ et $64 \mathrm{~mm}$ et deux de longueur $L$ égale à $128 \mathrm{~mm}$ (en tout onze éprouvettes).

3. 2 RÉSUltATS DES MESURES. - $\beta$ et $\gamma$ étant liés par (24), notre modèle théorique et en particulier la relation (25), donne les variations du rapport $S(\beta)=$ $R_{\mathrm{b}}(\beta) / R_{\mathrm{b}}(0)$. Pour expliciter $\beta$ en fonction des paramètres caractérisant une éprouvette, il faut d'abord remarquer que deux parties serties, et non pas une comme dans un balai, interviennent. Ensuite, en notant $\rho$ la résistance du matériau constituant les filaments et en combinant (6) et (24), on obtient, compte tenu du facteur deux :

$$
\beta=\frac{D}{\sqrt{L}} \sqrt{\frac{f R_{\mathrm{s}}}{2 L_{\mathrm{A}} \phi \rho}} .
$$

( $\left.{ }^{1}\right)$ Ce type de mesure serait peut-être possible pour les filaments situés à la périphérie, mais, presqu'à coup sûr, impossible pour ceux situés près de l'axe (au cœur) de l'éprouvette. 
La relation (27) montre que $\beta$ est une fonction de $L$, dont on est maître expérimentalement, et, dans le cas où ce paramètre peut varier, de $R_{\mathrm{s}}$. La détermination de rapports expérimentaux : $R_{\text {exp. }}(L) /$ $R_{\text {idéale }}(L)$ peut donc permettre d'exploiter le modèle théorique et de mettre en évidence l'influence plus ou moins grande de $R_{\mathrm{s}}$. Nos résultats expérimentaux sont représentés par des carrés pleins sur la figure 6 . Une courbe, obtenue d'une manière qui sera précisée au paragraphe 3.4 donne l'allure générale du phénomène, qui est une décroissance du rapport $R_{\text {exp. }} / R_{\text {idéale }}$ en fonction de $L$. Comme on peut le remarquer, les points expérimentaux, correspondant à une même longueur, présentent une certaine dispersion. Cette dispersion n'est pas due aux erreurs de mesures, qui, du fait de la qualité des matériels employés (source de courant très bien régulée et voltmètre digital) sont très faibles, mais à la nature même des éprouvettes dont on mesure les résistances. Parmi les effets pouvant conduire à cette dispersion, on peut citer, par exemple, une légère transposition par l'intermédiaire de laquelle, certains filaments situés à la périphérie, à une extrémité, se retrouvent plus près de l'axe, à l'autre.

On peut dire également, que dans le domaine de mesure considéré $(0<I<50 \mathrm{~A})$ les différentes résistances mesurées sont indépendantes du courant

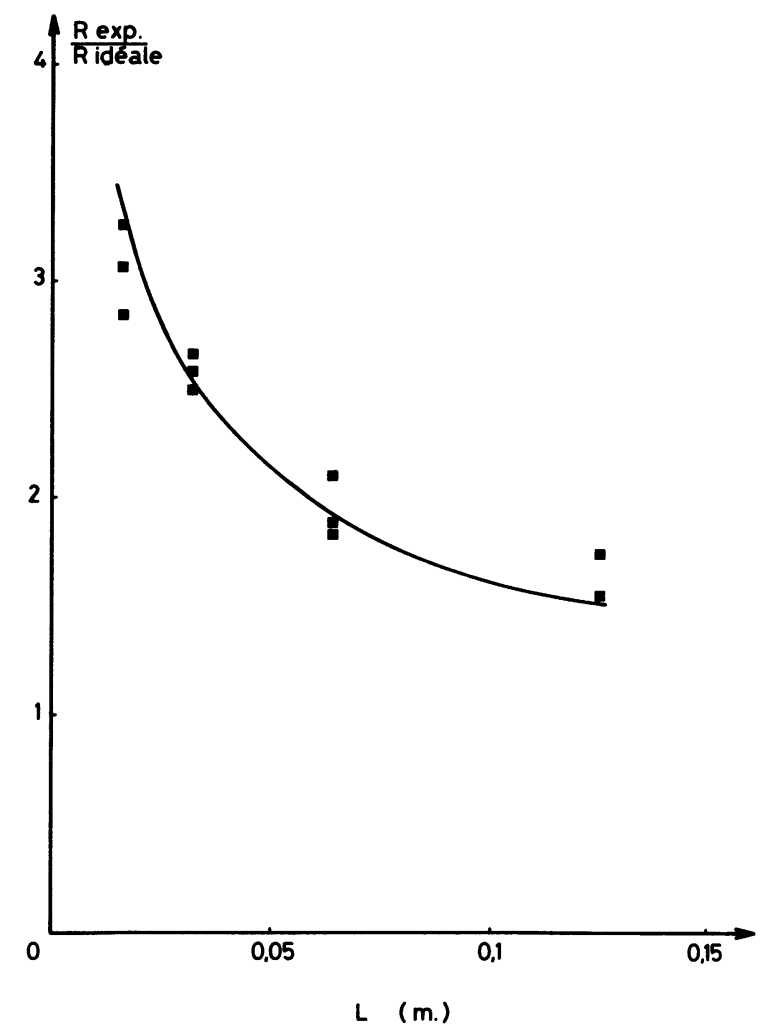

Fig. 6. - Valeurs expérimentales du rapport $R_{\text {exp. }} / \boldsymbol{R}_{\text {idéale }}$ correspondant à des éprouvettes de différentes longueurs.

[Experimental values of the ratio $R_{\text {exp. }} / R_{\text {ideal }}$ corresponding to test samples of various lengths.]
( $V$ proportionnel à $I)$. Si un tel type de non-linéarité existait ( $V$ fonction non linéaire de $I)$, il ne pourrait être dû qu'aux caractéristiques tunnel des interfaces filament/filament, principalement constituées d'oxyde cuivreux $\left(\mathrm{Cu}_{2} \mathrm{O}\right)$. Or, une jonction tunnel $\mathrm{Cu} / \mathrm{Cu}_{2} \mathrm{O}$ stoechiométrique/Cu possède une hauteur de barrière $V_{\mathrm{b}}$ de l'ordre de 1,2 $\mathrm{V}$ pour les électrons [14], ce qui est très important devant les tensions que nous mesurons $(V<0,05 \mathrm{~V})$. Comme, par ailleurs, on sait [15] que lorsque $V \ll V_{\mathrm{b}}$ la caractéristique $V(I)$ d'une jonction tunnel est linéaire, on en déduit que, dans notre cas, les interfaces filament/filament doivent avoir un comportement du même type.

3.3 DéTERMINATION DE $R_{s}$. - Si notre modèle s'appliquait de façon idéale, les parties serties, correspondant aux différentes éprouvettes, devraient être caractérisées par la même résistance surfacique $R_{\mathrm{s}}$. Notre modèle donne, à partir de (27), l'expression suivante pour ce paramètre :

$$
R_{\mathrm{s}}=\frac{2 L_{\mathrm{A}} \phi \rho}{D^{2} f} \beta^{2} L
$$

Avec les valeurs numériques, exprimées en unités M.K.S.A., relatives à nos éprouvettes, et en prenant un coefficient de foisonnement égal à $\pi / 2 \sqrt{3}$ (sections circulaires des filaments tangentes) l'expression (28) s'écrit :

$$
R_{\mathrm{s}}=7,42 \times 10^{-10} \beta^{2} L \quad \Omega \cdot \mathrm{m}^{2} .
$$

On peut, à partir de (29), déterminer les valeurs expérimentales de $R_{\mathrm{s}}$. En effet, pour une valeur de $R_{\text {exp. }} / R_{\text {idéale }}$ correspondant à une longueur $L$ donnée, on détermine, à l'aide de la figure 4 , la valeur $\beta_{\text {exp. }}$. de $\beta$. Cette valeur correspond à l'abscisse du point de la courbe $S(\beta)$ qui a pour ordonnée $S=R_{\text {exp. }} / R_{\text {idéale }}$. Ensuite, il suffit de multiplier le nombre figurant dans (29) $\operatorname{par} \beta_{\text {exp. }}^{2}$. . On obtient, ainsi, les différentes valeurs reportées sur la figure 7 . Ces résultats permettent de déterminer la valeur moyenne de $\beta_{\text {exp. }} L^{1 / 2}$ : 0,796 que nous utiliserons ultérieurement.

Avant de commenter ces résultats, il convient de remarquer que l'expression de la résistivité radiale moyenne $\rho_{\mathrm{r}}$ du matériau composite, représenté en coupe sur la figure $2 \mathrm{~b}$ (alternance de couches d'épaisseur $\phi$, de résistivité nulle, et de couches infiniment fines de résistance surfacique $R_{\mathrm{s}}$ ) s'écrit :

$$
\rho_{\mathrm{r}}=\frac{R_{\mathrm{s}}}{\phi} \text {. }
$$

$\mathrm{Si}$ on considère alors les valeurs expérimentales extrêmes de $R_{\mathrm{s}}$ (voir Fig. 7), on observe que $\rho_{\mathrm{r}}$ calculé à l'aide de (30), varie entre des limites :

$$
6,06 \times 10^{-6} \Omega . \mathrm{m}<\rho_{\mathrm{r}}<1,45 \times 10^{-5} \Omega . \mathrm{m}
$$

qui sont plus de deux ordres de grandeurs au-dessus de la valeur de la résistivité du matériau constituant 


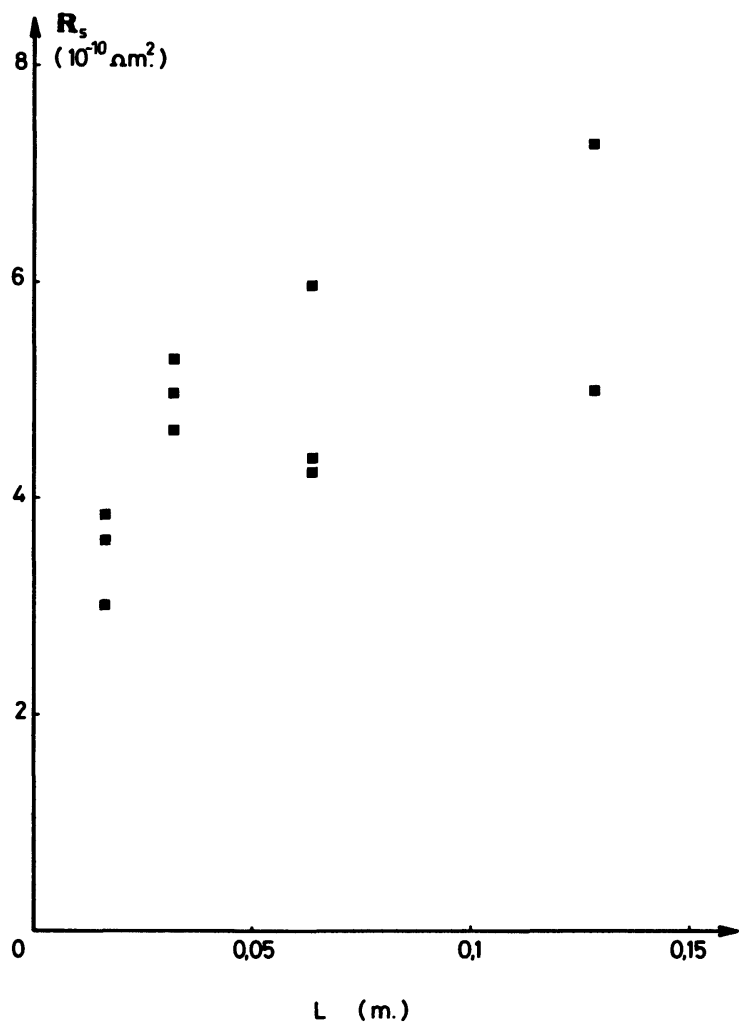

Fig. 7. - Valeurs expérimentales de $R_{\mathrm{s}}$ en fonction de $L$. [Experimental values of $R_{\mathrm{s}}$ against $L$.]

les filaments $\left(\rho_{\text {CuCd }}=2,02 \times 10^{-8} \Omega . \mathrm{m}\right)$. Cela indique bien que le "matériau " constituant la partie sertie et représenté en coupe sur la figure 1 a une résistivité électrique fortement anisotrope. Cette grande valeur de la résistivité radiale est due, d'une part, à un compactage insuffisant, et d'autre part, au fait que les filaments comportent une couche d'oxyde d'environ $5 \mathrm{~nm}$ d'épaisseur, dont l'effet isolant peut subsister après le sertissage.

Ensuite on remarque que les valeurs extrêmes de $R_{\mathrm{s}}$ sont dans un rapport légèrement supérieur à deux et que $R_{\mathrm{s}}$ a tendance à augmenter avec $L$. Nous pensons que ce dernier point ne constitue pas une mise en défaut de notre modèle, mais qu'il est lié à l'existence de points de contact, entre les fibres, en dehors des parties serties. En effet, plus les éprouvettes sont courtes, moins les filaments ont la possibilité de s'épanouir, donc d'être isolés les uns des autres, entre les parties serties; ce qui est contraire à une de nos hypothèses. Dans ces conditions, pour les éprouvettes courtes, il doit se produire une redistribution du courant entre les parties serties. Nos résultats expérimentaux conduisent donc, dans ce cas, à des valeurs de $\beta$, donc de $R_{\mathrm{s}}$, plus faibles que celles que l'on aurait si les filaments étaient réellement isolés les uns des autres.

3.4 Comparaison DE $R_{\text {exp. }} / R_{\text {idéale }}$ ET DE $S(\beta)$. La relation (27) indique de façon explicite que si $R_{\mathrm{s}}$ est constant, $\beta$ varie comme $L^{-1 / 2}$. Il est donc intéressant d'étudier la variation du rapport $R_{\text {exp. }} / R_{\text {idéale }}$ en fonction de $L^{-1 / 2}$. Pour effectuer une comparaison de ce rapport avec la fonction $S(\beta)$, nous avons porté, sur la figure 8 , les points expérimentaux et la fonction $S\left(0,796 L^{-1 / 2}\right)$. Le nombre 0,796 , correspond, comme nous l'avons déjà précisé, à la valeur moyenne de $\beta_{\text {exp. }} L^{1 / 2}$. L'examen de la figure 8 montre que la courbe théorique est assez proche des points expérimentaux, pour les faibles valeurs de $L^{-1 / 2}$ (donc pour les grandes valeurs de $L$ ) et au-dessus des points correspondant à la plus petite valeur de $L$. Ceci est en accord avec notre précédente remarque sur l'effet perturbateur de contacts interfilaments, entre les sertissages, pour les faibles valeurs de $L$. C'est à partir de points de la courbe $S\left(0,796 L^{-1 / 2}\right)$ que nous avons tracé celle de la figure 6 .

\section{Conclusion.}

Une étude théorique au premier ordre et une étude expérimentale du mode de répartition du courant dans un balai multifilamentaire ont été réalisées. On a montré, grâce à un modèle, que cette répartition du courant (continu) est analogue à ce que l'on rencontre avec l'effet de peau, dans un matériau massif parcouru par un courant alternatif. Dans le cas d'un balai multifilamentaire réalisé par sertissage, la répartition du courant s'effectue, à partir de la périphérie

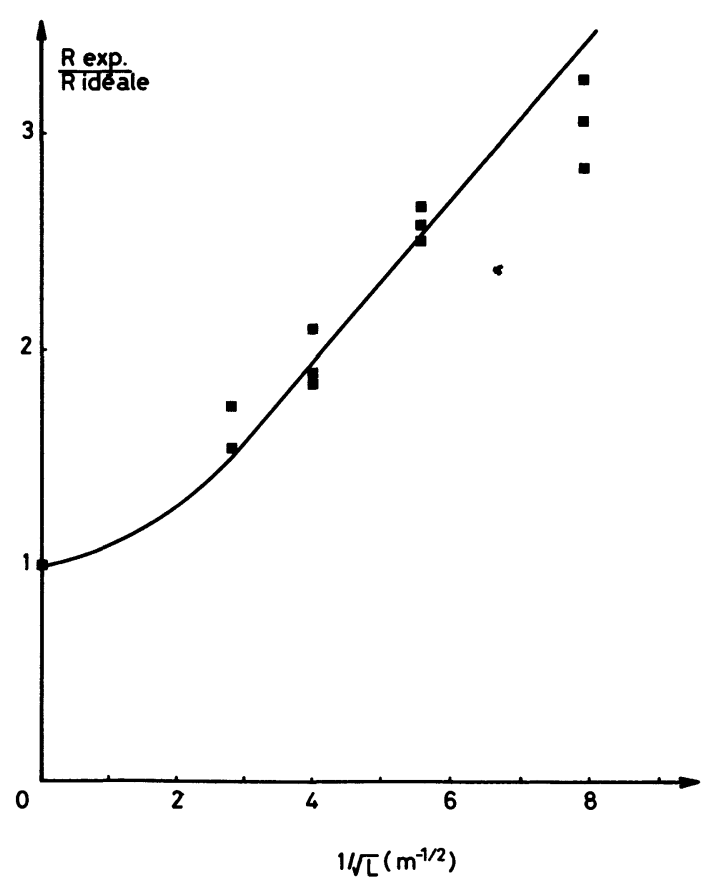

Fig. 8. - $\square$ Valeurs expérimentales du rapport $R_{\text {exp. }} / R_{\text {idéale }}$ en fonction de $L^{-1 / 2}$. Graphe de $S(\beta)=R_{\text {exp. }} / R_{\text {idéale }}$ avec $\beta=0,796 L^{-1 / 2}$.

[ Experimental values of the ratio $R_{\text {exp. }} / R_{\text {ideal }}$ against $L^{-1 / 2}$. Plot of $S(\beta)=R_{\text {exp. }} / R_{\text {ideal }}$ with $\beta=0.796 L^{-1 / 2} .7$ 
de la partie sertie, par un cheminement de filament à filament. Dans ces conditions, les filaments situés à l'extérieur sont les « premiers servis » et transportent donc plus de courant que ceux situés à l'intérieur. Cet effet se traduit par une augmentation de la résistance du balai par rapport à ce que l'on aurait dans le cas d'un sertissage parfait. Le modèle permet de mettre en évidence le paramètre qui détermine la qualité (bonne ou mauvaise) de la répartition du courant. Une série de mesures sur des éprouvettes de longueurs différentes, comportant un sertissage à chacune des extrémités, a permis de vérifier la validité du modèle.

Cette étude s'inscrit dans le cadre plus général d'une recherche sur les contacts électriques glissants multifilamentaires, dont l'objectif est de déterminer entre autres, les origines de la d.d.p. de contact que l'on observe avec de tels dispositifs. Nous avons vu que la répartition inégale du courant, entre les filaments, constitue une de ces origines. Les mesures que nous effectuons sur des balais frottant sur des pistes, montrent que la nature, physico-chimique, de l'interface filament/piste a, aussi, une grande importance. Par ailleurs, il faut savoir que pour un contact électrique glissant, les pertes se décomposent en une partie électrique et une partie mécanique, liée au frottement. Nos résultats expérimentaux montrent que quand un filament frotte sur une piste, dans des conditions déterminées, les pertes mécaniques qu'il engendre dépendent très peu du passage du courant. Sur le plan de l'optimisation globale d'un balai multifilamentaire, on a donc intérêt à ce que tous les filaments participent de façon égale au passage du courant. Une campagne de mesure sur des balais comportant 750 filaments (au lieu de 3000 ) vient de démarrer. Nous pensons pouvoir évaluer, dans les mois à venir l'avantage réel que l'on peut tirer d'une meilleure répartition du courant.

\section{Remerciements.}

Cette étude a été effectuée dans le cadre d'un contrat D.R.E.T. et grâce au soutien du GRECO 40 « Contacts Electriques ». L'auteur exprime sa gratitude envers J. P. Chabrerie et le Professeur G. Fournet pour de fructueuses discussions ainsi qu'à L. Huaumé pour son assistance technique.
[1] McNAB, I. R., Recent advance in electrical current collection, Wear 59 (1980) 259-276.

[2] ReICHNER, P., High current tests of metal brushes, Proc. 26th Annu. Holm Conf. on Electrical Contacts, Chicago, II, 1980, in IEEE Trans. Components, Hybrid Manuf. Technol., (March 1981) $2-4$.

[3] Adkins, C. M. and Kuhlmann-Wilsdorf, D., Development of High-Performance Metal Fiber Brushes Part III, Electrical Contacts, 1980 (III. Inst. Techn. Chicago, II) 1980 , p. 67-72.

[4] Lupton, W. H. and Reichner, P., Ultrahigh speed fiber brush design and tests, Wear 78 (1982) 139149.

[5] Ross, D. P., Ferrentino, G. L. and Young, J. F., Experimental determination of the contact friction for an electromagnetically accelerated armature, Wear 78 (1982) 189-200.

[6] Hwang, B. H., Singh, B., Vook, R. W. and Zhang, J. G., In situ Auger electron spectroscopy characterization of wet- $\mathrm{CO}_{2}$-lubricated sliding copper electrical contacts, Wear 78 (1982) 7-16.

[7] Singh, B., Zhand, J. G., Hwang, B. H. and Vook, R. W.., Microstructural characterization of rotating $\mathrm{Cu}-\mathrm{Cu}$ electrical contacts in vacuum and wet $\mathrm{CO}_{2}$ environnements, Wear 78 (1982) 17-28.
[8] Boyer, L., Chabrerie, J. P. and Saint-Michel, J., Low wear metallic fiber brushes, Wear 78 (1982) 59-68.

[9] Boyer, L. and Chabrerie, J. P., Current collection in a homopolar machine using metallic fiber brushes, 1983, International Current Collection Conference, Octobre 1983, Annapolis, Maryland (U.S.A.).

[10] Edison. Th. A., Electrical Generator and Motor, U.S. Patent $\mathrm{n}^{\circ} 276$ 233, April 24, 1883.

[11] Chabrerie, J. P., Brevet français (ANVAR-DRET) $\mathrm{n}^{\mathrm{o}}$ 29716, Sept. 1977.

[12] Boyer, L., Chabrerie, J. P., Saint-Michel, J., Brevet français (DRET) no 8211897 , Juillet 1982.

[13] Holm, R., Electric Contacts (Springer Verlag) 4e édition, 1967.

[14] Assimos, J. A. and Trivich, D., Photovoltaic properties and barrier heights of single-crystal and polycristalline $\mathrm{Cu}_{2} \mathrm{O}-\mathrm{Cu}$ contacts, J. Appl. Phys. 44 (1973) 1687.

[15] Simmons, J. G., Generalized Formula for the Electric Tunnel Effect between Similar Electrodes separated by a Thin Insulating Film, J. Appl. Phys. 34 (1963) 1793. 